\title{
Progress in intermolecular pinacol cross coupling methodologies
}

\author{
Bruna S. Terra ${ }^{a}$ and Fernando Macedo Jr. ${ }^{b_{*}}$ \\ ${ }^{a}$ Departamento de Ciências Farmacêuticas, Universidade Estadual de Londrina; \\ ${ }^{b}$ Departamento de Química, Universidade Estadual de Londrina, \\ PO Box 6001, Londrina - PR 86051-990, Brazil \\ E-mail: macedofc@uel.br
}

\begin{abstract}
The reductive carbonyl coupling leading to 1,2-diols, known as pinacol coupling, is conceptually the most direct approach that leads to this structural pattern. From the nineties, exciting developments have resulted in efficient protocols to the coupling of two different carbonyl compounds mixed in approximately equimolar amounts. This article reports the progress that has been made so far and the challenges existing in the methods and their general synthetic application to the construction of complex bioactive substances.
\end{abstract}

Keywords: 1,2-Diols, low-valent metals, reductive carbonyl coupling, single electron transfer

\section{Table of Contents}

1. Introduction

2. Seminal Work

3. Pinacol Cross Coupling Methodologies

4. Applications in the Synthesis of Bioactive Substances

5. Conclusions

6. References

\section{Introduction}

The reductive carbonyl coupling leading to 1,2-diols, known as pinacol coupling, is one of the oldest and best-studied of carbon-carbon bond-forming reactions (Scheme 1).

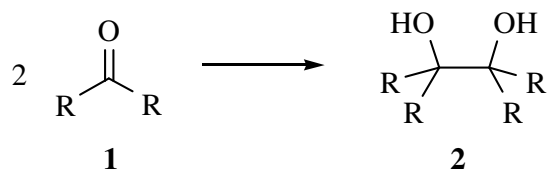

Scheme 1. Pinacol coupling reaction. 
The 1,2-diol unit is one of the most ubiquitous functional groups in bioactive substances and the pinacol coupling has been playing an important role in the synthesis of pharmacologically important agents (e.g. taxol, ${ }^{1}$ cotylenol, ${ }^{2}$ HIV-I protease ${ }^{3}$ ). Other important approaches to vicinal diols, such as epoxide ring opening and olefin dihydroxylation, require the preexistence of the central carbon-carbon bond in the starting materials.

Since its first report, a number of reproducible and high yielding protocols were described. ${ }^{4}$ Virtually all of them involve the use of metals in a low oxidation state acting in single electron transfer (SET) processes.

The recognition of the basic factor that influences the stereochemical outcome of the reaction permitted a high level of control in the distribution of diasteroisomeric adducts. More recently, enantioselective approaches have been developed for the successful dimerization of carbonyl compounds. Efficient catalytic methodologies have also been proposed. All these developments however, are to much of an extent, limited to intramolecular coupling or to homocoupling of an intermolecular reaction. These aspects are very properly discussed in an excellent recent reviews. ${ }^{5}$

Comparatively, there is many fewer reports of intermolecular cross coupling pinacol reaction. Despite of the introduction of some promising methodologies developed for this aim, the efficient and wide scoping cross fashion of the reaction remains a great challenge. This account concerns to the efforts, advances and challenges involved in pinacol cross coupling reaction of two different carbonyl components.

\section{Seminal Work}

Scheme 2 shows the basic accepted mechanism of pinacol coupling promoted by low-valent metals. The first step consists in the formation of a ketyl radical by the electron transfer from the metal to carbonyl compound. The subsequent $\mathrm{C}-\mathrm{C}$ bond forming step could takes place from two metal-bonded ketyl radicals or through a pseudo-bridged intermediate in an intramolecular fashion. When the dimerization of two such radicals operates through a pseudo-bridged metal atom, the threo selectivity in the product predominates, due to steric reasons. On the other hand, when two such species couple through a non-bridged intermediate, the formation of the erythro product is favoured. ${ }^{5}$

Under these mechanistic backgrounds, the efficiency of cross coupling of two different carbonyls with similar reduction potentials is intrinsically limited. Thus, good yields of desired mixed 1,2-diols could not be expected unless in cases where one of the two carbonyls components is electronically activated or when an excess of one of them is employed. 


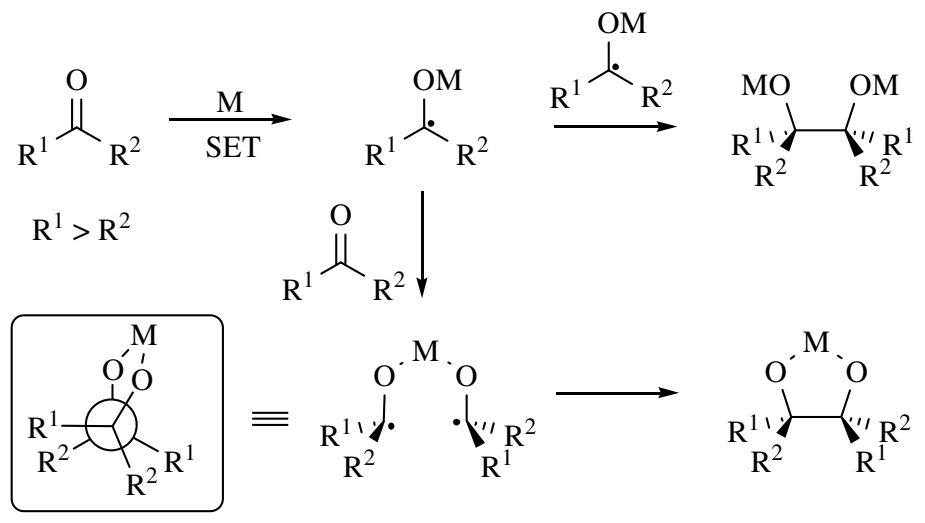

Scheme 2. Basic mechanism of pinacol coupling reaction.

In general, a trend toward statistical distribution of three possible pinacols has been reported from binary mixtures $(\mathrm{A} / \mathrm{B})$ of aldehydes and ketones under reductive coupling conditions. However, systematic investigation by Mundy and co-workers using equimolar amounts of different ketones pairs in the presence of $[\mathrm{Mg}-\mathrm{Hg}] / \mathrm{TiCl}_{4}$ showed that the statistically expected distribution: A-B / A-A / B-B (2:1:1), is not always observed. ${ }^{6}$ Thus, the synthetic use of the reaction could not be reliably based on these expectations. In this study the authors have demonstrated that steric and stereoelectronic factors related to the structure of the substrates may lead to unpredictable proportions of products. In a reductive coupling reaction with cyclohexanone, for example, cyclopentanone (B) furnished the expected pinacol mixture in an almost statistical distribution (Scheme 3). On the other hand, 2- and 4-methylcyclohexanone showed comparatively greater resistance to homocoupling.

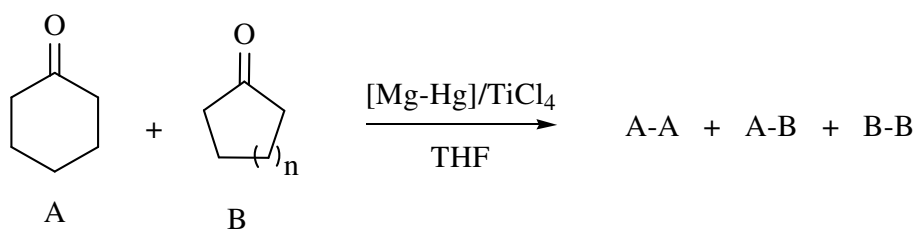

Scheme 3. Systematic investigation of distribution of pinacol coupling products.

Some authors have showed that unsymmetrical coupling adducts can be synthesized in useful yields if one carbonyl component is used in excess. Obviously, such an approach is only synthetically practical in cases where an excess of one inexpensive carbonyl component is involved and preferably if its self-coupling by-product could also be easily removed.

In the context of natural product synthesis, $\mathrm{Corey}^{7}$ explored this concept in the obtaining carbonyl coupling products using Ti-based reductive mixtures (Scheme 4). 


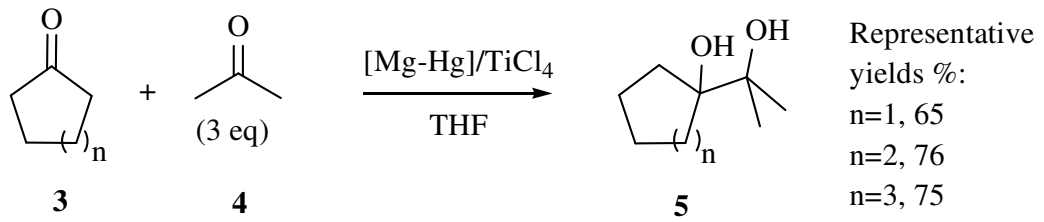

Scheme 4. Ti-based reductive carbonyl cross coupling.

This reductive condition did not give good results with low molecular weight aldehydes and, in these cases, the reagent formed by reaction of cyclopentadienyltitanium trichloride and lithium aluminum hydride was indicated. In another work $^{8}$, a titanocene-based reductive system $\left(\mathrm{Cp}_{2} \mathrm{TiCl}_{2} / \mathrm{Mn}\right)$ was also used to promote pinacol coupling between acetophenone and a large excess of acetone resulting in the corresponding cross-coupling product, 2,3-dihydroxy-2methyl-3-phenylbutane, in $83 \%$ yield.

McMurry and co-workers ${ }^{9}$ have showed that low-valent titanium reagents prepared from $\mathrm{TiCl}_{3}$ are also effective to promote reductive carbonyl coupling. ${ }^{4}$ Although McMurry's protocols leads to deoxygenated coupling products, the intermediacy of pinacolate species is widely accepted and the 1,2-diols can be isolated when reaction is carried out at $0{ }^{\circ} \mathrm{C}$. They observed that a particularly efficient cross coupling was obtained when diaryl ketones were treated with acetone and $\mathrm{Li} / \mathrm{TiCl}_{3}$ (Scheme 5).

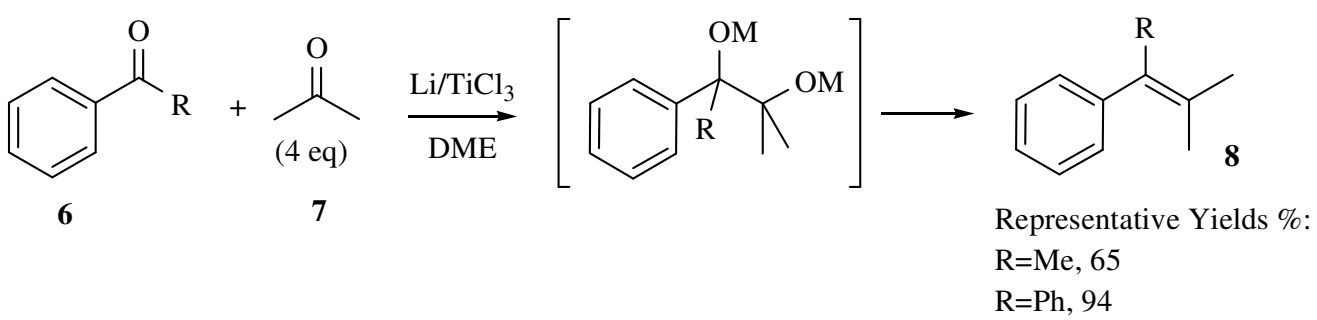

Scheme 5. Cross coupling with aryl ketones.

The high yields of the mixed product were preserved even when acetone and benzophenone were reacted in equimolar amounts $(81 \%)$. Because of the tendency of diaryl ketones to form anion radicals faster than acetone, higher levels of benzophenone self-coupling should be expected. Thus, the authors considered unlikely that the mixed pinacol reaction of these components is in fact a radical coupling process. Alternatively, they proposed a mechanism in which the stabilized radical anions derived from diaryl ketones are rapidly reduced further to dianions that couple in a mixed way with acetone by nucleophilic addition (Scheme 6). 

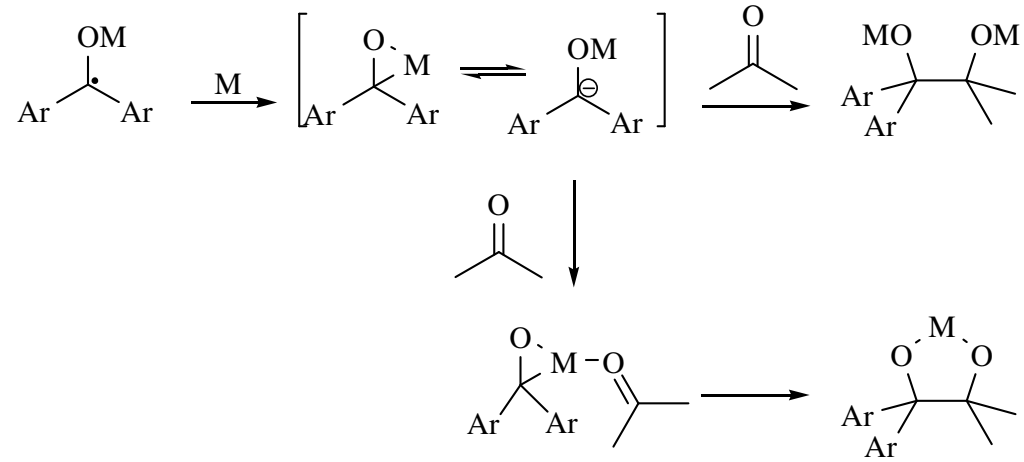

Scheme 6. Involvement of nucleophilic intermediates in pinacol coupling reaction mediated by transition metals.

This result is one of the first evidence that in the case of transition metals, the metal insertion into the carbonyl group can generate an anionic metaloxirane that can attack another carbonyl compound.

\section{Pinacol Cross Coupling Methodologies}

In 1989 Pedersen et al. showed in two independent articles that the known dimeric vanadium complex, $\left[\mathrm{V}_{2} \mathrm{Cl}_{3}(\mathrm{THF})_{6}\right]_{2}\left[\mathrm{Zn}_{2} \mathrm{Cl}_{6}\right]$ could efficiently cross couple two different aldehydes mixed in equimolar amounts. ${ }^{10 \mathrm{a}, \mathrm{b}}$ Similar to McMurry's interpretation in the case of diaryl ketones, Pedersen et al. assumed that any ketyl radical generated upon reaction with one vanadium(II) center might be rapidly reduced further by the second vanadium(II) leading to an organometallic intermediate (Scheme 7). In a preliminary study involving dimerization reaction, Pedersen and co-workers have demonstrated that the vanadium complex couples aryl aldehydes in higher yields and faster than non-functionalized aliphatic aldehydes. Aryl or dialkyl ketones give little or no coupling products under the same conditions.

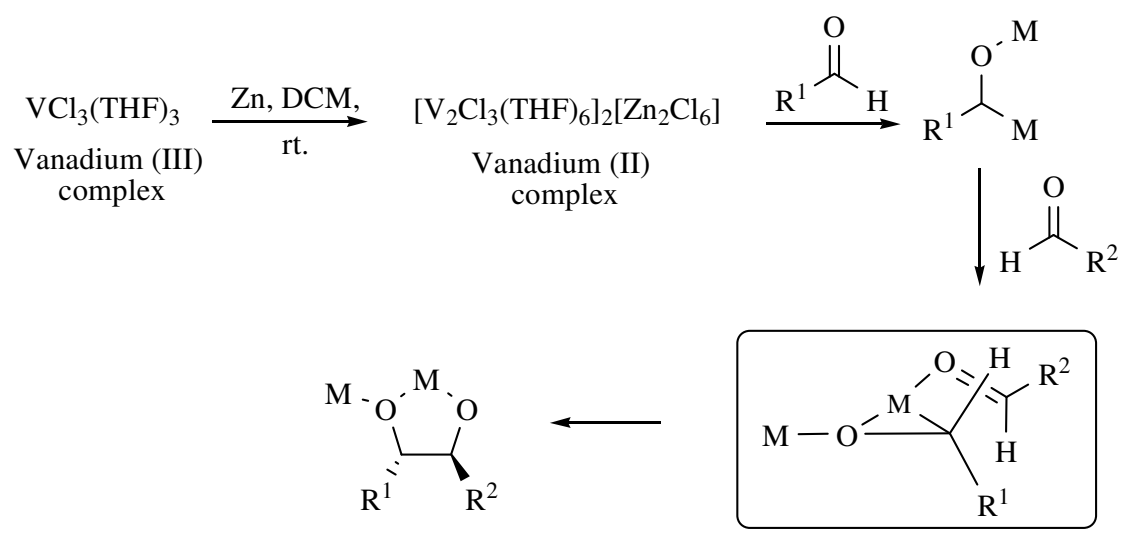

Scheme 7. Proposed mechanism for the pinacol coupling mediated by vanadium (II). 
In this way, the cross coupling of a non-aryl aldehyde and an aryl aldehyde was possible by a slow addition of the aromatic partner to a solution of vanadium complex and 1.0 equivalent of the less reactive carbonyl aldehyde (Scheme 8$).{ }^{10 a}$
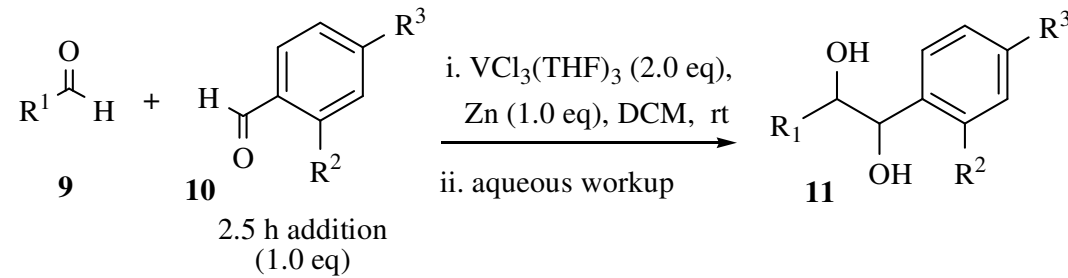

$$
\begin{aligned}
& \text { Representative yields \% (threo/erythro } d r \text { ): } \\
& \mathrm{R}^{1}={ }^{\mathrm{n}} \mathrm{C}_{5} \mathrm{H}_{11} ; \mathrm{R}^{2}=\mathrm{R}^{3}=\mathrm{H}, 49(1: 1) \\
& \mathrm{R}^{1}={ }^{\mathrm{n}} \mathrm{C}_{5} \mathrm{H}_{11} ; \mathrm{R}^{2}=\mathrm{H} ; \mathrm{R}^{3}=\mathrm{Br}, 25(1: 1) \\
& \mathrm{R}^{1}={ }^{\mathrm{n}} \mathrm{C}_{5} \mathrm{H}_{11} ; \mathrm{R}^{2}=\mathrm{Br} ; \mathrm{R}^{3}=\mathrm{H}, 82(1: 1) \\
& \mathrm{R}^{1}={ }^{\mathrm{n}} \mathrm{C}_{5} \mathrm{H}_{11} ; \mathrm{R}^{2}=\mathrm{H} ; \mathrm{R}^{3}=\mathrm{CF}_{3}, 77(1: 1) \\
& \mathrm{R}^{1}={ }^{\mathrm{n}} \mathrm{C}_{5} \mathrm{H}_{11} ; \mathrm{R}^{2}=\mathrm{CF}_{3} ; \mathrm{R}^{3}=\mathrm{H}, 79(1: 1) \\
& \mathrm{R}^{1}={ }^{\mathrm{i}} \mathrm{Pr} ; \mathrm{R}^{2}=\mathrm{CF}_{3} ; \mathrm{R}^{3}=\mathrm{H}, 72(4: 1)
\end{aligned}
$$

Scheme 8. Pinacol cross coupling of aryl aldehyde and non-aryl aldehyde mediated by vanadium (II).

In these studies, the cross coupling reactions of an aryl aldehyde with an ortho or para electron withdrawing group gave the best results. This trend was rationalized as a consequence of the lowered reduction potential of these substrates.

Less reactive aryl aldehydes furnished best results when used as their corresponding dimethyl acetals 13 (Scheme 9).

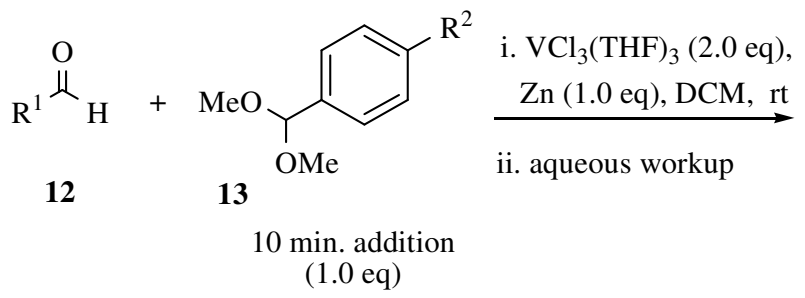<smiles>[R]c1ccc(C(OC)C([R])O)cc1</smiles>

14
Representative yields \% $(d r)$

$\left[\mathrm{R}^{1}=\mathrm{Ph}\left(\mathrm{CH}_{2}\right)_{2}\right]$ :

$\mathrm{R}^{2}=\mathrm{H}, 75(1: 1)$

$\mathrm{R}^{2}=\mathrm{OMe}, 78(1: 1)$

Scheme 9. Pinacol cross coupling of non-aryl aldehyde and dimethyl acetals mediated by vanadium (II).

It was also observed that the rate of coupling might be accelerated if the aldehyde contained an appropriately placed chelating group, particularly if six- or seven-membered chelate rings with a vanadium center could be formed (chelation-accelerated aldehydes or CA aldehydes). ${ }^{10 \mathrm{~b}}$

In this way, the cross coupling of equimolar mixtures of two electronically similar non-aryl aldehydes was possible by a slow addition of a chelating aldehyde to a solution of vanadium complex and a 1.0 equivalent of a less reactive aldehyde. In this respect, 3 -formylpropanamides $\mathbf{1 6}$ proved to be good substrates as illustrated in Scheme 10. 


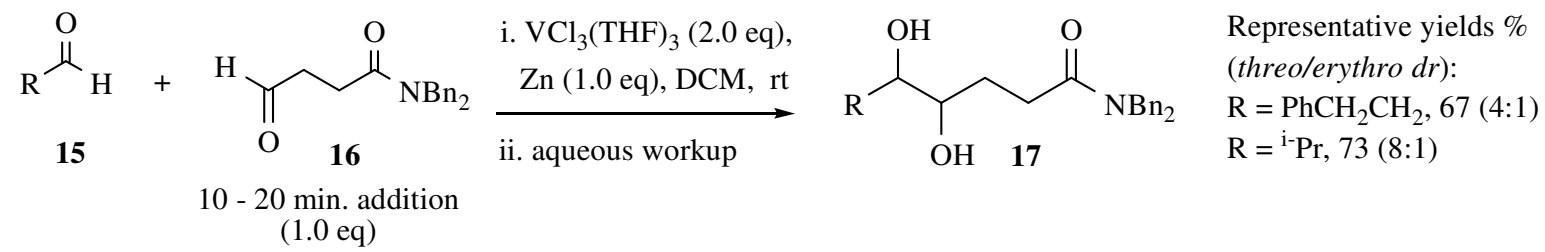

Scheme 10. Pinacol cross coupling of two non-aryl aldehydes mediated by vanadium (II).

Based on the important role played by the amide function in the above investigations, Cozzi and co-workers investigated the insertion of a chiral amide group aiming at the development of the enantioselective pinacol of Pedersen's methodology (Scheme 11). The cross coupling of a chiral aromatic aldehyde $\mathbf{1 9}$ with aliphatic aldehydes afforded threo diols in high diastereoisomeric ratio and in $75-84 \%$ enantiomeric excess. ${ }^{11 a, b}$

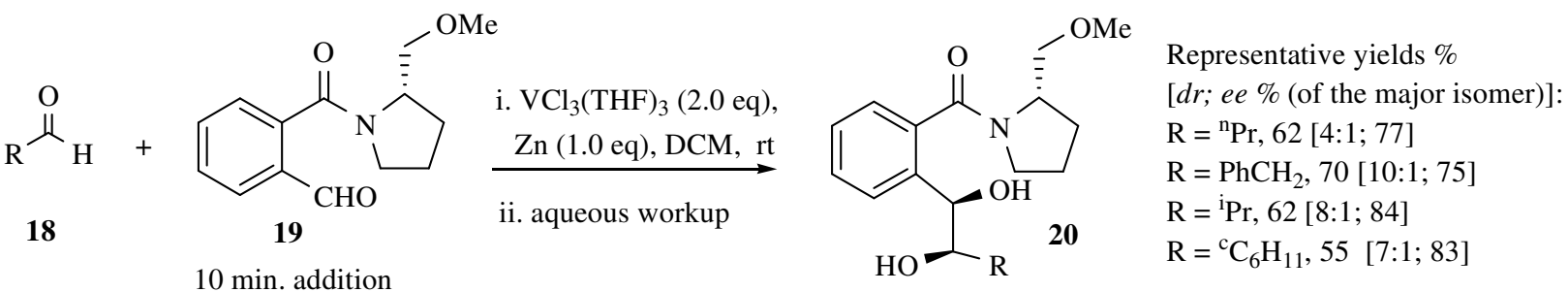

Scheme 11. Pinacol cross coupling of chiral formylamides with aliphatic aldehydes mediated by vanadium (II).

The enantiomeric excesses were further improved with the use of a pyrrolidine bearing a bulkier residue at C-2. The change in the chiral auxiliary led to an increase in the ee's to values generally higher than $90 \%$, while the yields and diastereoselectivity was affected at a lower extent. $^{12}$

$\mathrm{N}$-Alkoxycarbonyl-2-aminoaldehydes $\mathbf{2 2}$ proved also to be good candidates for this reaction and were employed successfully to prepare 3-amino-1,2-diols $\mathbf{2 3}$ via pinacol cross coupling reaction with aliphatic aldehydes. ${ }^{13 a, b}$

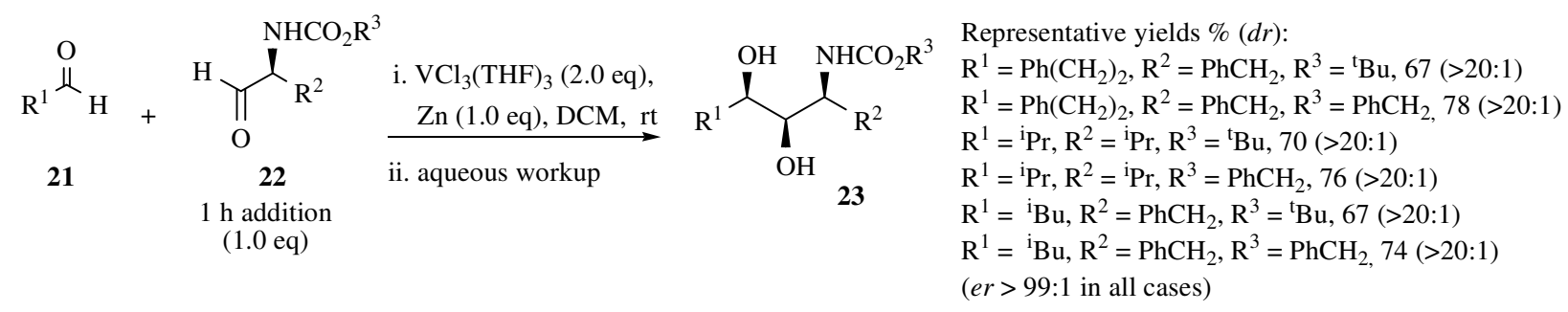

Scheme 12. Pinacol cross coupling of N-alkoxycarbonyl-2-amino aldehydes mediated by vanadium (II). 
It was found that branching of the aliphatic aldehydes (R1) and several functional groups in the side chains (R2) of the 2-[N-(alkoxycarbonyl)amino] aldehydes do not impair the cross coupling reactions (Scheme 12). In most cases, good yields of a single isomer was obtained $(d r>$ 20:1). The effect of the $N$-alkoxycarbonyl group (R3) on the yield of the cross coupling reaction could be generalized: $\mathrm{MeO}_{2} \mathrm{C}>$ Alloc $>\mathrm{Cbz}>$ Boc.

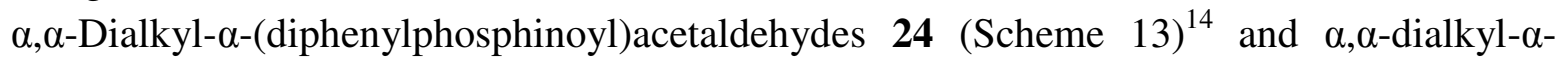
formylpropionates 27 (Scheme 14) ${ }^{15}$ were other classes of compounds that could be coupled in a mixed way with saturated aldehydes in good yields. To all these cases (22, 24 and 27) a chelation control, as showed in Scheme 13, was suggested to rationalize the stereochemical outcome.

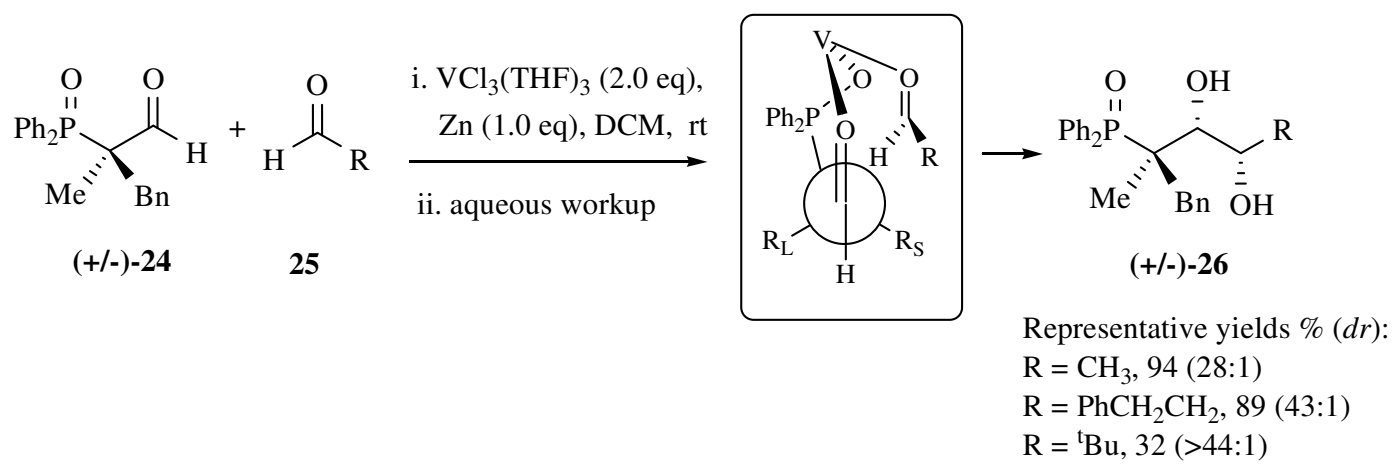

Scheme 13. Cross coupling of $\alpha, \alpha$-dialkyl- $\alpha$-(diphenylphosphinoyl)acetaldehydes mediated by vanadium (II).

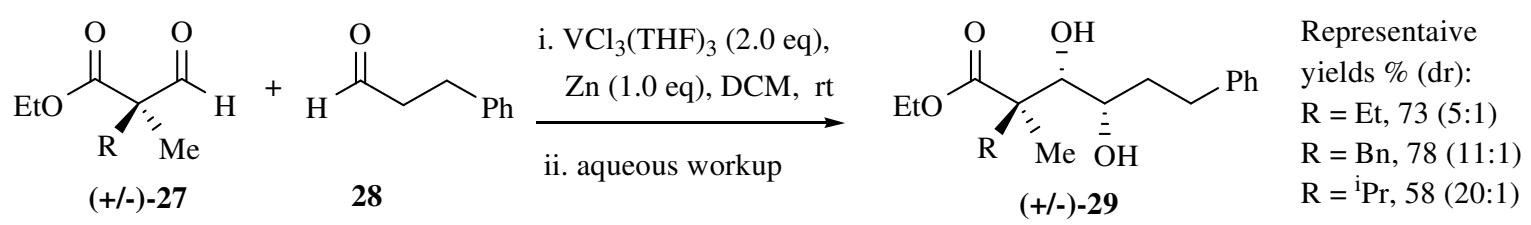

Scheme 14. Cross coupling of $\alpha, \alpha$-dialkyl- $\alpha$-formylpropionates mediated by vanadium (II).

Curiously, the slow addition of the hindered chelating aldehydes to a solution of the vanadium (II) reagent and the nonchelating aldehyde was not required in these cases. Considering that one equivalent of the $\mathrm{CA}$ aldehyde can form a chelate with $\left[\mathrm{V}_{2} \mathrm{Cl}_{3}(\mathrm{THF})_{6}\right]_{2}\left[\mathrm{Zn}_{2} \mathrm{Cl}_{6}\right]$, the author argued that cross-coupling is presumably favored because the less hindered, nonchelating aldehyde effectively competes with a second equivalent of the hindered CA aldehyde for coordination to the same metal center. ${ }^{14}$ That assumption is supported by the fact that homocoupling of CA aldehydes becomes competitive with cross-coupling when a hindered nonchelating aldehyde like pivaldehyde is employed.

The high diastereofacial selectivity observed in both cases was rationalized from a Cram chelated type model in which the nonchelating aldehyde coordinates and reacts with the least hindered face of the chelating aldehyde. 
The same vanadium complex was also used successfully in a practical method for the direct hydroxymethylation of aldehydes and ketones via pinacol cross coupling with paraformaldehyde. ${ }^{16}$ The use of a large excess of paraformaldehyde (at least 10 equiv.) was essential for the formation of terminal diols at high yields (Scheme 15). This protocol was assumed to be more general than the formylation reaction employing reductive coupling chemistry of aqueous titanium (III) reagents.

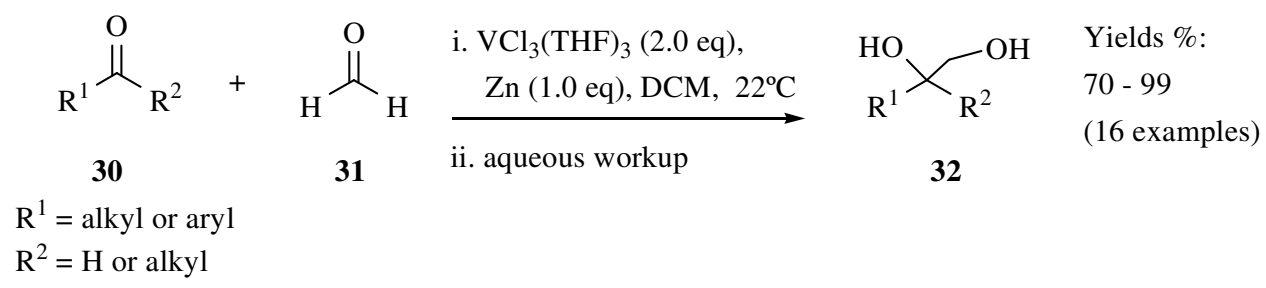

Scheme 15. Hydroxymethylation of aldehydes and ketones mediated by vanadium (II).

Fujiwara and co-workers reported the cross-coupling reactions of diaryl ketones in which the carbonyl group carbon reactivity in diaryl ketones is reversed by lanthanoid metals to nucleophilic. ${ }^{17}$ The addition of ketones to the diaryl ketone-ytterbium mixture at room temperature gives the corresponding cross-coupling products in high yields (Scheme 16). The authors have demonstrated by IR that the participation of the intermediate $\mathbf{3 4}$ is dependent of the stoichometry ration between $\mathrm{Yb}$ metal and diaryl ketone. Upon treatment of benzophenone with 1.0 equiv or excess amount of $\mathrm{Yb}$ metal, they observed, before hydrolysis, a strong peak at 1566 $\mathrm{cm}^{-1}$ assignable to 34. When 0.5 equiv of $\mathrm{Yb}$ metal was used, it was not verified and, in this case, it was proposed the formation of diols through cyclization of a pseudo-bridged intermediate as depicted in Scheme 2.

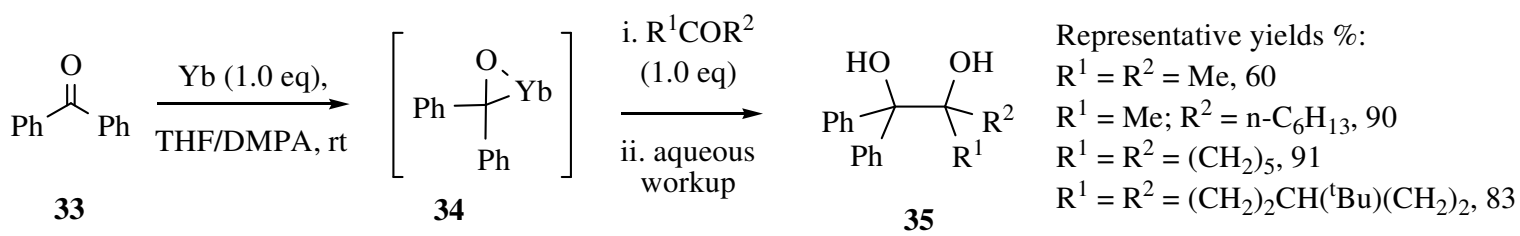

Scheme 16. Pinacol cross coupling mediated by ytterbium.

Askham and Carrol developed an efficient route to unsymmetrical vicinal diols by reaction of thermally stable zirconium oxiranes with aromatic aldehydes and ketones. ${ }^{18}$ The addition of 37 to one equivalent of an aromatic aldehyde or ketone proceeded readily, producing, after acidic hydrolysis, the threo vicinal diols as the favored isomers (Scheme 17). 

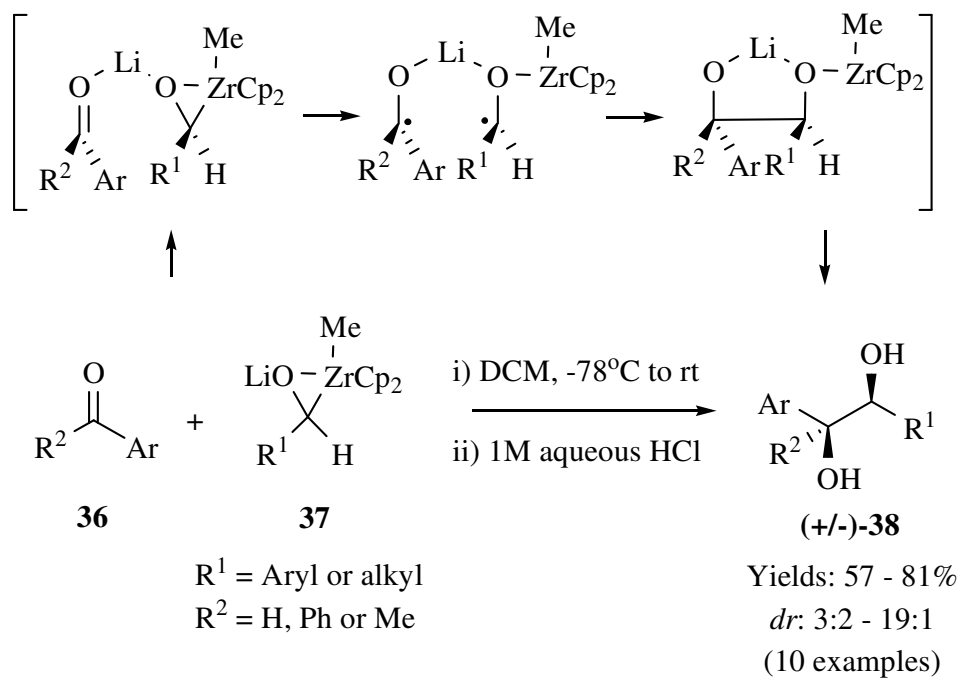

Scheme 17. Cross coupling of zirconium oxiranes and aromatic aldehyde or ketones.

Aliphatic aldehydes or ketones failed as the electrophilic partner in this reaction. The dependence on the presence of an aromatic functionality was rationalized as a requirement of the reduction of the electrophile to a ketyl radical in the rate-determining step.

In the proposed mechanism, coordination to the lithium cation would lower the reduction potential of the carbonyl compound facilitating the subsequent SET step. This interaction would also maintain the two $\mathrm{C}-\mathrm{O}$ bonds approximately parallel as the two carbons approach each other. Minimization of steric interactions in such an intermediate would then lead to the threo product.

Yus and collaborators ${ }^{19}$ have showed that the lithiation of different diary1 ketones with an excess of lithium and a catalytic amount of naphthalene leads to the formation of the corresponding dianions which eact with aldehydes and ketones to give the mixed pinacol adducts as show in Scheme 18.

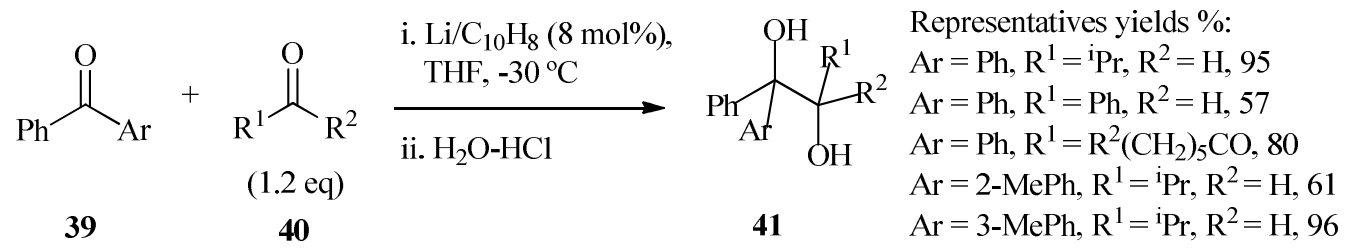

Scheme 18. Cross coupling of diaryl ketones and aldehydes or ketones mediated by lithium.

It has been demonstrated that a suspension of samarium(II) dibromide prepared from $\mathrm{Sm}_{2} \mathrm{O}_{3}$ is able to mediate efficiently reductive coupling of ketones and aldehydes. ${ }^{20}$ Attempts to crosscouple aliphatic ketones under these conditions have led to almost statistical mixtures of diols. In the case of the reaction between benzophenone and other ketones, mixed in equimolar amounts, diphenyl diols have been selectively obtained (Scheme 19). It is noteworthy that camphor and benzophenone alone do not dimerize, but they combine each other to give mixed pinacols. 


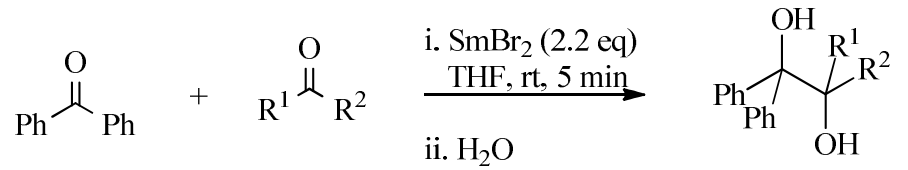

$$
\begin{aligned}
& 42 \\
& \text { 43a) 2-octanone } \\
& \text { 43b) camphor } \\
& \text { 44c) methone }
\end{aligned}
$$

Scheme 19. Cross coupling of ketones mediated by $\mathrm{SmBr}_{2}$.

Takabe and co-workers ${ }^{21}$ showed that $N$-alkylated phthalimides $\mathbf{4 5}$ underwent reductive cross-coupling reactions with aliphatic ketones or aldehydes mediated by samarium(II) diiodide to provide $\alpha$-hydroxylactams in a moderate and good yield (Scheme 20).<smiles>[R]N1C(=O)c2ccccc2C1=O</smiles>

45<smiles>[R]C([R])=O</smiles>

46<smiles>[R]N1C(=O)c2ccccc2[C@]1(O)C([R])([R])O</smiles>

47

Representative yields \%:

$\mathrm{R}=\mathrm{CH}_{3}, \mathrm{R}^{1}=\mathrm{CH}_{3} \mathrm{CH}_{2}, \mathrm{R}^{2}=\mathrm{H}, 60$

$\mathrm{R}=\mathrm{CH}_{2} \mathrm{Ph}, \mathrm{R}^{1}=\mathrm{CH}_{3} \mathrm{CH}_{2}, \mathrm{R}^{2}=\mathrm{H}, 79$

$\mathrm{R}=\mathrm{CH}\left(\mathrm{CH}_{3}\right)-1-$ Naphythyl, $\mathrm{R}^{1}=\mathrm{CH}_{3} \mathrm{CH}_{2}, \mathrm{R}^{2}=\mathrm{H}, 44$

$\mathrm{R}=\mathrm{CH}_{2} \mathrm{Ph}, \mathrm{R}^{1}=\mathrm{CH}_{3}, \mathrm{R}^{2}=\mathrm{CH}_{3}, 43$

Scheme 20. Cross coupling of $N$-alkylated phthalimides mediated by $\mathrm{SmI}_{2}$.

This protocol proved to be ineffective if $N$-H and $N$-phenylphthalimides or non-aromatic $N$ alkylimides are used.

Low-valent chromium is another metal that has been received much attention and, in the last 20 years, a number of studies on its use in pinacol cross coupling reactions has been reported. Takai was the first to show that $\mathrm{Cr}(\mathrm{II})$, in the presence of iodinetrimethylsilane, is effective to couple acrolein acetals $\mathbf{4 8}$ with aldehydes in a cross fashion for the generation of 1,2-diol derivatives (Scheme 21). ${ }^{22}$

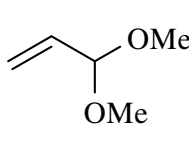

48<smiles>[R]C(C=O)OC</smiles>

(+/-)-49

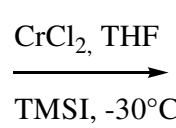

TMSI, $-30^{\circ} \mathrm{C}$<smiles>[R]C(OC)C(O)C(C=C)OC</smiles>

(+/-)-50

Scheme 21. Pinacol cross coupling of acrolein acetals and aldehydes mediated by chromium(II).

More recently Takai reported pinacol cross-coupling reactions between vinyl ketones and aldehydes by the use of a excess of chromium chloride and chlorotriethylsilane as a scavenger (Scheme 22). ${ }^{23 a, b}$ 


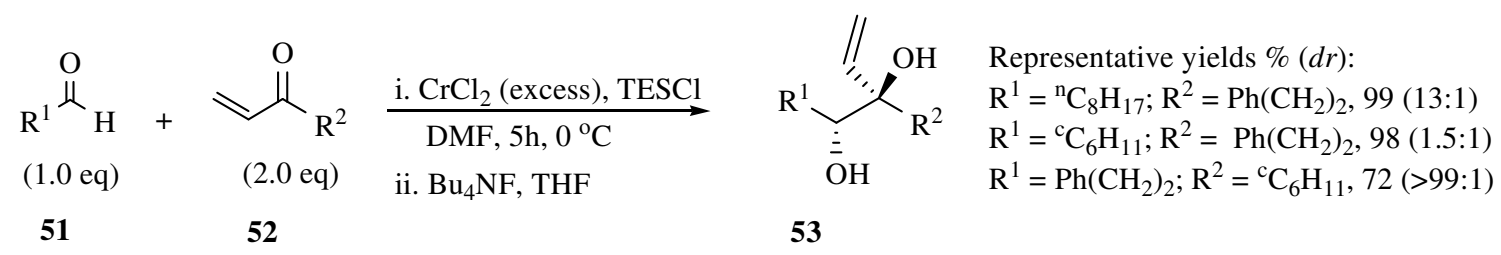

Scheme 22. Pinacol cross coupling of vinyl ketones and aldehydes mediated by chromium(II).

Based on the Takai chromium-mediated pinacol cross coupling and on the methodology developed by Fürstner for a catalytic Nozaki-Hiyama reaction, Boeckmann developed the first catalyzed reductive carbonyl cross coupling protocol. In this protocol, acetals of acrolein or methacrolein and aldehydes were efficiently coupled in the presence of chlorotrimethylsilane and stoichiometric amounts of manganese as reducing agent. ${ }^{24}$ Sodium iodide was incorporated to the mixture in the optimized conditions. The alcohols were obtained generally in excellent yield and good selectivity in favor of the anti isomer (Scheme 23).

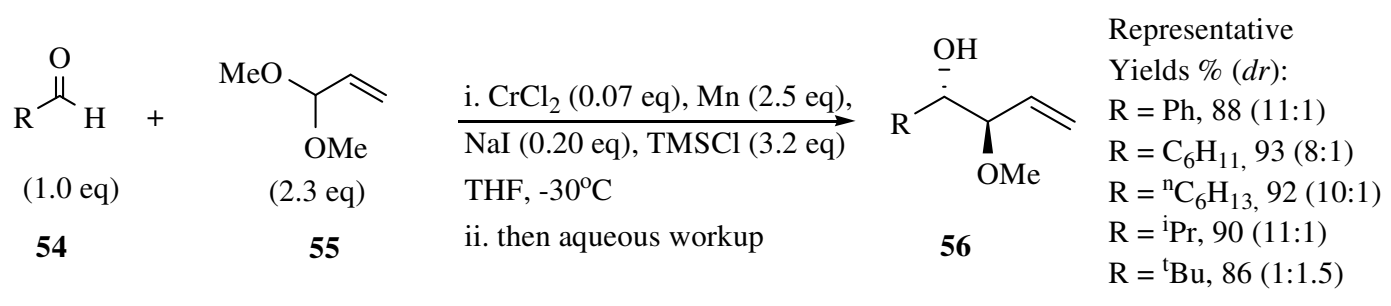

Scheme 23. Chromium catalyzed pinacol cross coupling of acetals of acroleins and aldehydes.

Groth and co-workers extended the method to pinacol cross coupling reaction of $\alpha, \beta-$ unsaturated carbonyl compounds and aldehydes to form 1,2-diols using $10 \mathrm{~mol} \%$ of chromium. ${ }^{25 a, b, c}$ Vinyl ketones (Scheme 24) and acroleins (Scheme 25) were coupled with aldehydes in moderate to good yields and with variable diastereoselectivity.

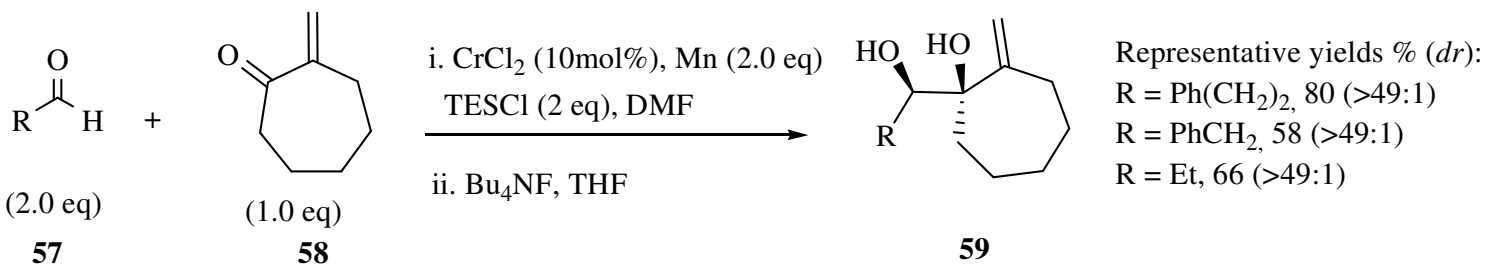

Scheme 24. Chromium catalyzed pinacol cross coupling of vinyl ketones and aldehydes.

In general, the homo-coupling of vinyl ketones have not exceeded 5\%. On the other hand the proportions of dimerization increased in the reaction between aromatic aldehydes lowering the yields to $25 \%$. 


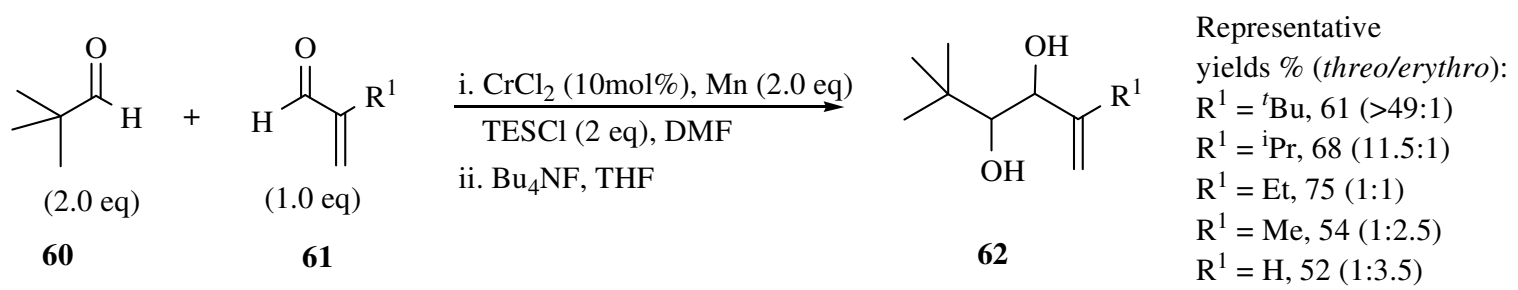

Scheme 25. Chromium catalyzed pinacol cross coupling of acroleins and aldehydes.

From a green chemistry perspective, the development of protocols that uses substoichiometric amounts of transition metals or even metal free methodologies is very attractive. Additionally, synthetic methodologies working in aqueous media are also particularly desirable.

Miyoshi and collaborators ${ }^{26}$ have developed an efficient methodology to coupling diketones and aldehydes in the presence of bismuth trichloride and metallic zinc to afford the corresponding $\alpha, \beta$-dihydroxyketones with diastereoselectivities ranging from 2:1 and 6:1 approximately in favor of the erythro isomers (Scheme 26). Although these results are given for THF the reaction takes place even in aqueous media. It was also found that the present reaction proceeds smoothly by using a catalytic amount of bismuth trichloride with some lost in yields and stereoselectivity. Additionally, a preliminary result demonstrated the potential to explore this methodology also to $\alpha$-ketoesteres.

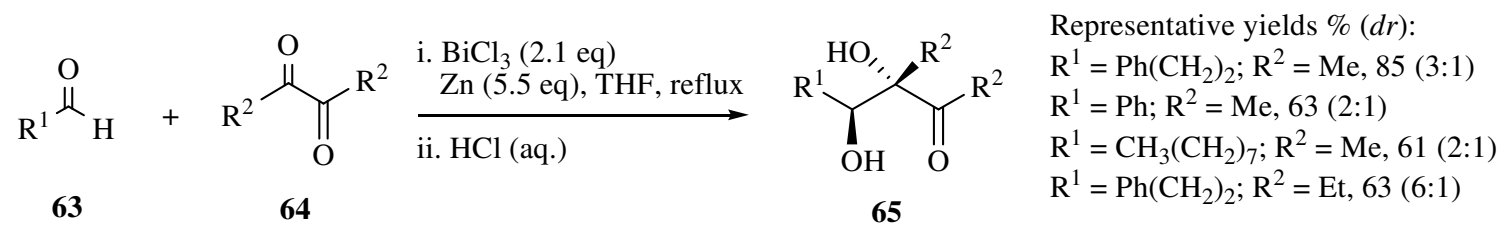

Scheme 26. Pinacol cross coupling of 1,2-diketones and aldehydes mediated Bismuth.

It was demonstrated that magnesium promotes cross coupling of aromatic ketones and other aliphatic carbonyl component resulting in unsymmetrical vicinal diols in moderate yields (Scheme 27). ${ }^{27}$ Although the protocol requires a large excess (10 equivalents) of the aliphatic carbonyl partner, symmetrical pinacols were not observed appreciably.

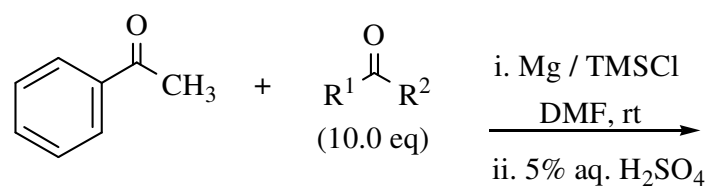

66
67

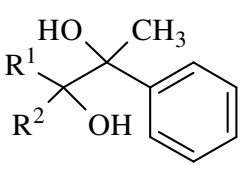

68
Representative yields \%:

$\mathrm{R}^{1}=\mathrm{Me} ; \mathrm{R}^{2}=\mathrm{H}, 44$

$\mathrm{R}^{1}={ }^{\mathrm{i}} \mathrm{Pr} ; \mathrm{R}^{2}=\mathrm{H}, 56$

$\mathrm{R}^{1}={ }^{\mathrm{t}} \mathrm{Bu} ; \mathrm{R}^{2}=\mathrm{H}, 64$

$\mathrm{R}^{1}=\mathrm{Me} ; \mathrm{R}^{2}=\mathrm{Me}, 70$

$\mathrm{R}^{1}=\mathrm{Me} ; \mathrm{R}^{2}=\mathrm{Et}, 32$

$\mathrm{R}^{1}=\mathrm{R}^{2}=-\left(\mathrm{CH}_{2}\right)_{4}^{-}, 44$

$\mathrm{R}^{1}=\mathrm{R}^{2}=-\left(\mathrm{CH}_{2}\right)_{5}^{-}, 49$

Scheme 27. Pinacol cross coupling mediated by magnesium. 
Nair and co-workers reported preliminary results of studies on the pinacol cross coupling reaction of aromatic aldehydes with chalcones in the presence of indium and indium trichloride (Scheme 28). ${ }^{28}$ Unlike alkali metals and most low-valent transition metals, indium is unaffected by water.
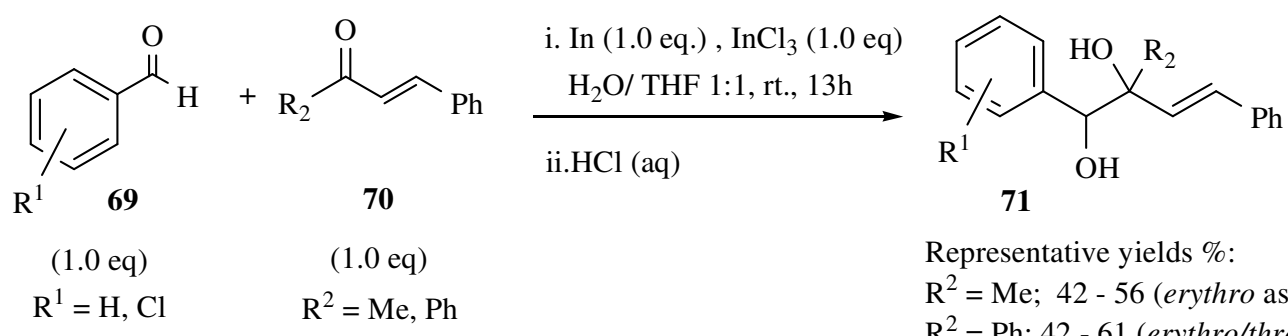

Representative yields \%:

$\mathrm{R}^{2}=\mathrm{Me} ; 42$ - 56 (erythro as single isomer)

$\mathrm{R}^{2}=\mathrm{Ph} ; 42-61$ (erythro/threo $\sim 2: 1$ )

Scheme 28. Pinacol cross coupling mediated by indium.

Recently, Loh and co-workers ${ }^{29}$ have reported a significant improvement in the scope of that approach showing that $\mathrm{Zn} / \mathrm{InCl}_{3}$ could lead to efficient pinacol cross-coupling reaction of aldehydes and aliphatic enones using the same solvent system (Scheme 29).

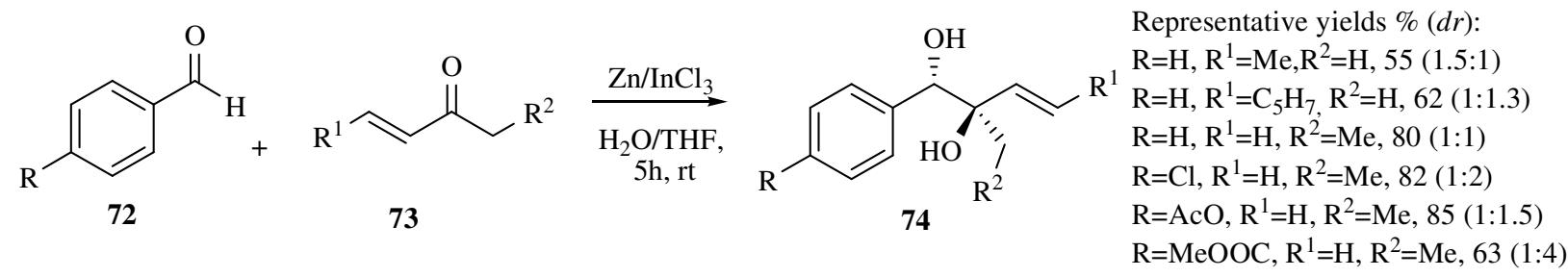

Scheme 29. Pinacol cross coupling mediated by indium.

Several different metal/salt combinations were investigated and $\mathrm{Zn} / \mathrm{InCl}_{3}$ was observed to be an effective system to furnish the cross coupling adducts in moderate to good yields. When other metals such as In, Al, Mg, Fe, and $\mathrm{Sn}$ were used, poor yields or no cross-coupled 1,2-diol products were observed. They showed that that the reduced metal( $(0)$ is indispensable and also that the pinacol coupling reaction using $\mathrm{Zn}(0)$ proceeded inefficiently in the absence of $\mathrm{InCl}_{3}$.

Additionally, the coupling of enones with aliphatic aldehydes showed to be promising using this protocol (Scheme 30).

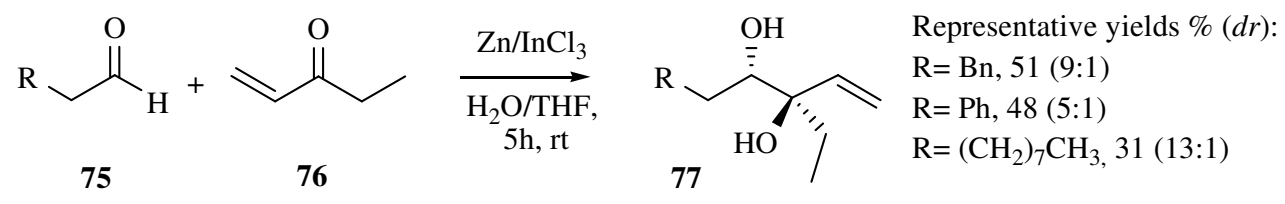

Scheme 30. Pinacol cross coupling mediated by indium. 
The use of electroreduction was report by Kise et $a .^{30}$ as an effective approach to cross coupling of aromatic ketones with aliphatic aldehydes in the presence of chlorotrimethylsilane and triethylamine by using a $\mathrm{Pb}$ cathode (Scheme 31). While the use of aldehyde gave corresponding unsymmetrical diols in high yields and low diastereoselectivity, ketones brought about poor results. Substitution of either electron-donating or electron-withdrawing group at the aromatic ring had little effect on the yields.

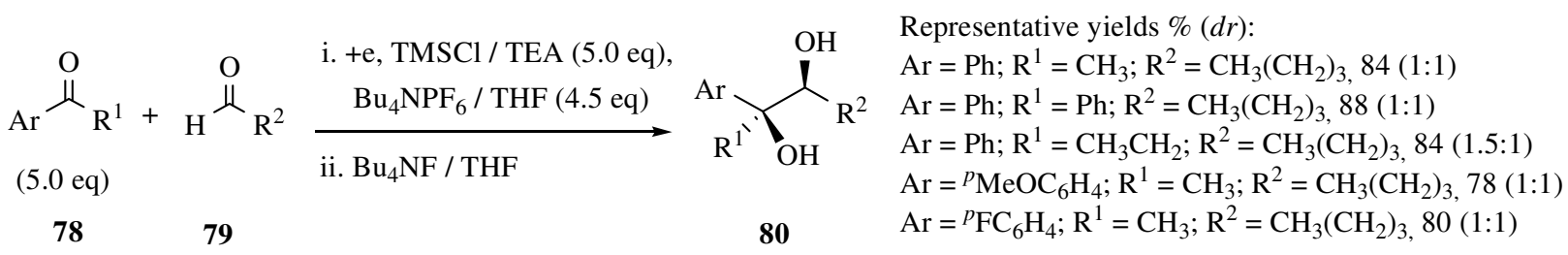

Scheme 31. Cross coupling of aromatic ketones with aliphatic aldehydes using electroreduction.

\section{Applications in the Synthesis of Bioactive Substances}

Pedersen demonstrated the synthetic utility of the methodology developed by him to the syntheses of sugars containing the 3-amino-1,2-diols subunit from $\mathrm{N}$-Cbz-(L)-aspartic acid. A model study was followed by the development of a synthesis of the amino sugar D-3-epidaunosamine, one diastereomer of a class of 2,3,6-trideoxy-3-aminohexoses that are important constituents of the antitumoral antibiotics daunomycin and adriamycin. In this case, slow addition of $\mathbf{8 2}$ (over $45 \mathrm{~min}$ ) to a cold dichloromethane solution of acetaldehyde gave (after aqueous work up) an 8:2:1 mixture of three diastereomers from which $\mathbf{8 3}$ was the major isolated cross-coupled product (Scheme 32). ${ }^{12 \mathrm{a}, \mathrm{b}}$

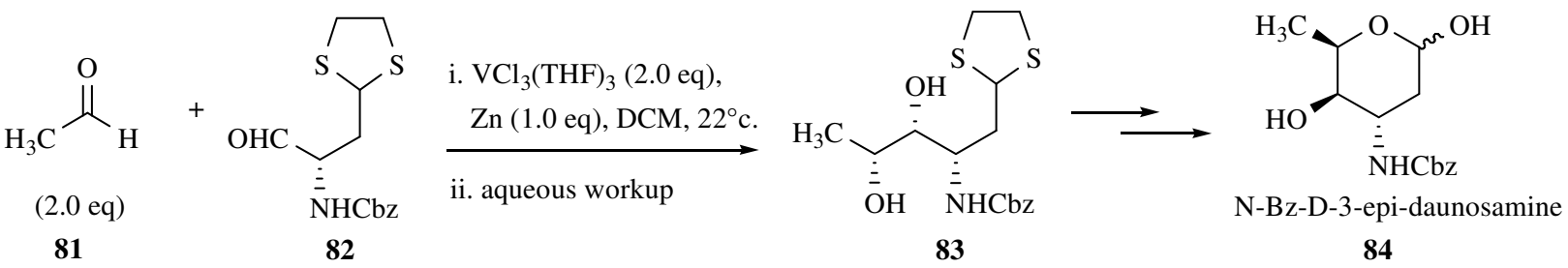

Scheme 32. Synthesis of an antitumor agent derivative.

Hodge used pinacol cross-coupling to synthesizes tricyclic ureas $\mathbf{8 8}$ that are active towards HIV-I protease. ${ }^{3}$ The coupling proceeded efficiently when a mixture of aldehyde $\mathbf{8 6}$ and the (D)phenylalaninal derivative $\mathbf{8 5}$ was treated with $\mathrm{VCl}_{3}(\mathrm{THF})_{3} / \mathrm{Zn}$ at room temperature for 2.5 hours. Under these conditions, the desired coupling product $\mathbf{8 7}$ was obtained in $84 \%$ yield and $85 \%$ diastereoselectivity (Scheme 33). 

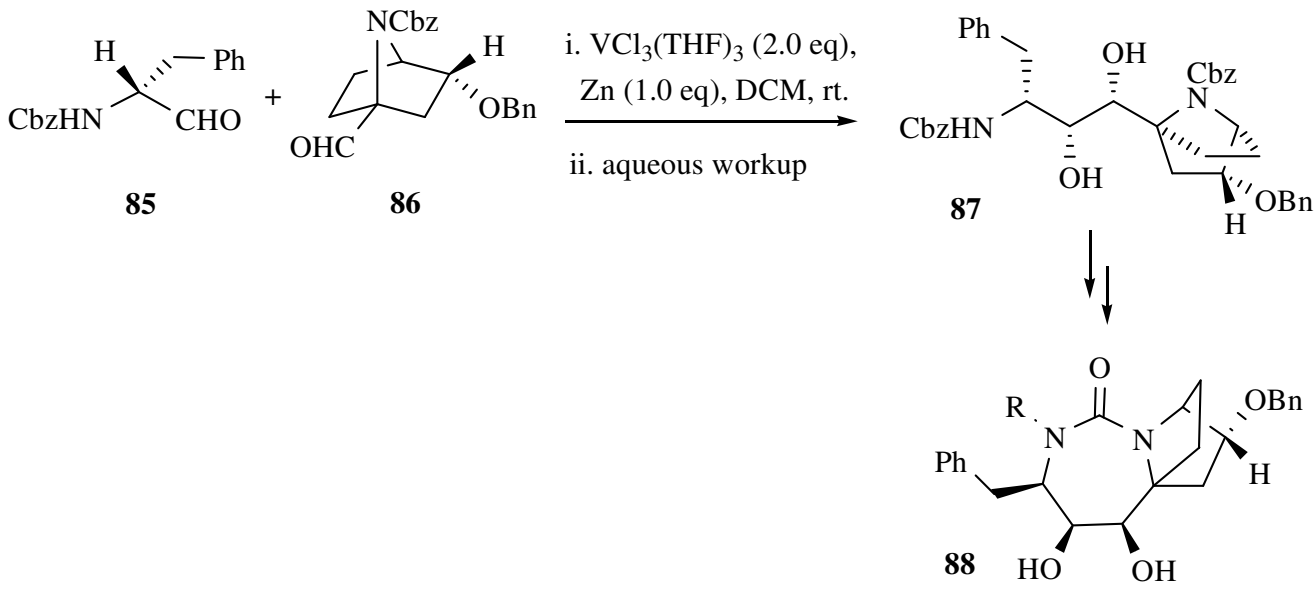

Scheme 33. Synthesis of HIV-I protease inhibitors.

Burger et al. ${ }^{31}$ reported a modified McMurry protocol to give unsymmetrically substituted diols. The reaction of 2,3-di-O-isopropylidene-D-glyceraldehyde $\mathbf{8 9}$ and methyl trifluoropyruvate 90 in the presence of $\mathrm{TiCl}_{3} / \mathrm{Zn}$ proceeded smoothly in moderate yield and with high stereoselectivity. The pinacol adduct 91 was further manipulated by a series of reactions to prepare 2-C-trifluoromethyl substituted D-ribose 92 (Scheme 34).

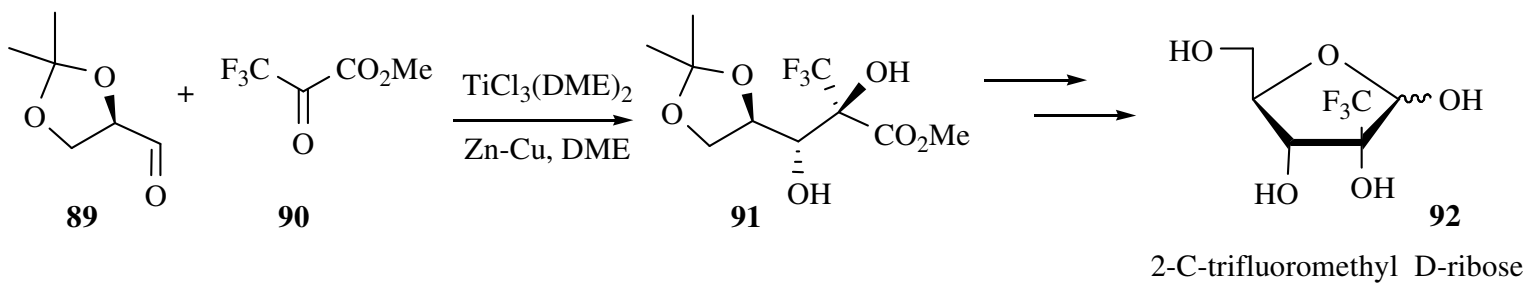

Scheme 34. Synthesis of 2-C-trifluoromethyl substituted D-ribose.

\section{Conclusion}

The intermolecular crossed version of pinacol coupling reaction is a very attractive route to complex pharmacologically active substances presenting the 1,2-diol motif. Exciting developments have resulted in high yielding protocols to the coupling of two different carbonyl compounds mixed in close to equimolar amounts. Nevertheless, even the most promising approaches are limited to the cases in which one of the carbonyl components is electronically activated or specifically functionalized to allow chelation to the metal and/or involve troublesome procedures. Therefore, a general and practical method for intermolecular pinacol cross coupling remains an inspiring challenge and constitutes a vast and opened field waiting to be explored. 


\section{References}

1. Mukaiyama, T.; Ogawa, Y.; Kuroda, K.; Matsuo, J. Chem. Lett. 2004, 33, 1412.

2. Kato, N.; Okamato, H.; Takeshita, H. Tetrahedron 1996, 52, 3921.

3. Han, W.; Pelletier, J.C.; Hodge, C.N. Bioorg. Med. Chem. Lett. 1998, 8, 3615.

4. McMurry, J.E. Chem. Rev. 1989, 89, 1513.

5. Chatterjee, A.; Joshi, N.N. Tetrahedron 2006, 62, 12137.

6. Mundy, B.P.; Srinivasa, R.; Kim, Y; Dolph, T.; Warnet, R.J. J. Org. Chem. 1982, 47, 1657.

7. Corey, E.J.; Danheiser, R.L.; Chandrasekaran, S. J. Org. Chem. 1976, 41, 260.

8. Paradas, M.; Campaña, A. G.; Estévez, R. E.; Cienfuegos, L. A.; Jiménez, T.; Robles, R.; Cuerva, J. M.; Oltra, J. E. J. Org. Chem. 2009, 74, 3616.

9. McMurry, J.E.; Krepski, L.R. J. Org. Chem. 1976, 41, 3929.

10. (a) Takahara, P.M.; Freudenberger, J.H.; Konradi, A.W.; Pedersen, S.F. Tetrahedron Lett. 1989, 30, 7177. (b) Freudenberger, J.H.; Konradi, A.W.; Pedersen, S.F. J. Am. Chem. Soc. 1989, $111,8014$.

11. (a) Annunziata, R.; Cinquini, M.; Cozzi, F.; Giaroni, P. Tetrahedron: Asymmetry 1990, 1, 355. (b) Annunziata, R.; Cinquini, M.; Cozzi, F.; Giaroni, P.; Benaglia, M. Tetrahedron 1991, 47, 5737.

12. Annunziata, R.; Benaglia, M.; Cinquini, M.; Cozzi, F.; Giaroni, P. J. Org. Chem. 1992, 57, 782.

13. (a) Konradi, A.W.; Pedersen, S.F. J. Org. Chem. 1990, 55, 4506. (b) Konradi, A.W.; Kemp S.J.; Pedersen, S.F. J. Am. Chem. Soc. 1994, 116, 1316.

14. Park, J.; Pedersen, S.F. J. Org. Chem. 1990, 55, 5924.

15. Min, K.; Park, J.; Pedersen, S. F. Synlett 1997, 1, 41.

16. Park, J.; Pedersen, S.F. Tetrahedron 1992, 48, 2069.

17. Hou, Z.; Takamine, K.; Aoki, 0.; Shiraishi, H.; Fujiwara, Y.; Taniguchi, H. J. Org. Chem. 1988, 53, 6077.

18. Askham, F.R.; Carroll, K.M. J. Org. Chem. 1993, 58, 7328.

19. Guijarro, D.; Mancheiio, B.; Yus, M. Tetrahedron 1993, 49, 1327.

20. Lebrun, A.; Namy, J-L.; Kagan, H. B. Tetrahedron Lett. 1993, 34, 2311.

21. Yoda, H.; Matsuda, K.; Nomura, H.; Takabe, K. Tetrahedron Lett. 2000, 41, 1775.

22. Takai, K.; Nitta, K.; Utimoto, K. Tetrahedron Lett. 1988, 29, 5263.

23. (a) Takai, K.; Morita, R.; Toratsu, C. Angew. Chem. Int. Ed. 2001, 40, 1116. (b) Takai, K.; Morita, R.; Matsushita, H.; Toratsu, C. Chirality 2003, 15, 17.

24. Boeckman, R.K.Jr.; Hudack, R.A.Jr. J. Org. Chem. 1998, 63, 3524.

25. (a) Groth, U.; Jung, M. Synlett 2002, 12, 2015. (b) Groth, U.; Jung, M.; Vogel, T. Chem. Eur. J. 2005, 11, 3127. (c) Fischer, S.; Groth, U.; Jung, M.; Lindenmaier, M.; Vogel, T. Tetrahedron Lett. 2005, 46, 6679.

26. Miyoshi, N.; Fukurna, T.; Wada, M. Chem. Lett. 1995, 999. 
27. Maekawa, H.; Yoshimasa, Y.; Shimada, H.; Yonemura, K.; Nishiguchi, I. Tetrahedron Lett. 2004, 45, 3869.

28. Nair, V.; Ros, S.; Jayan, C.N.; Rath, N.P. Tetrahedron Lett. 2002, 43, 8967.

29. Yang, Y.; Shen, Z.; Loh, T. Organic Lett. 2009, 11, 2213.

30. Kise, N.; Shiozawa, Y. Ueda, N. Tetrahedron 2007, 63, 5415.

31. Eilitz, U.; Böttcher, C.; Sieler, J.; Gockel, S.; Haas, A.; Burger, K. Tetrahedron 2001, 57, 3921.

\section{Authors' Biographies}

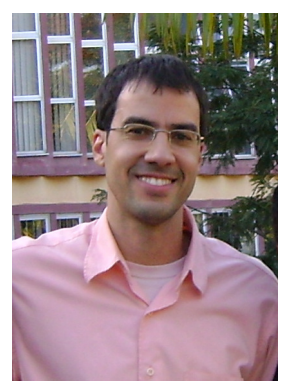

Fernando C. Macedo Jr was born in 1974. He graduated at Viçosa Federal University, Brazil, in 1998. He received his M.S. in chemistry from Campinas State University, Brazil, in 2001, and his Ph.D. in sciences from the same institution in 2004. After three years as post-doctoral fellow at Departament of Organic Chemistry, Bahia Federal University (Salvador, Brazil), he became adjunct professor at the Department of Chemistry, Londrina State University, Brazil. His research interests are in the fields of organic chemistry and NMR focusing in synthesis, structure, and bioactivity of natural products.

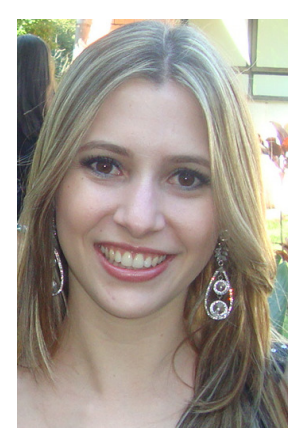

Bruna Silva Terra was born in 1988. She is an undergraduate student of pharmacy course at Londrina State University. She is currently developing research activities under the supervision of Dr. Fernando C. Macedo Jr. exploring the field of reductive carbonyl pinacol cross coupling. 